\title{
Residual Stresses Determination with Plasticity Effects by Electron Speckle-Interferometry Hole-Drilling Method
}

\author{
Leonid LOBANOV ${ }^{1,}$, , Viktor SAVITSKY ${ }^{1, b^{*}}$ \\ ${ }^{1}$ Paton Electric Welding Institute, the National Academy of Sciences of Ukraine, Kyiv, Ukraine \\ aholo@paton.kiev.ua, bviktor.savitsky@gmail.com
}

\begin{abstract}
Keywords: Residual Stresses, Speckle-Interferometry, Hole Drilling, ESPI-HD Method, Plasticity Effect
\end{abstract}

\begin{abstract}
The electron speckle-interferometry hole-drilling (ESPI-HD) method for determination of residual stresses (RS) is based on drilling of a blind hole with a diameter of $0.5-1.0 \mathrm{~mm}$ and calculating stresses using displacements data measured by a speckle-interferometer. According to the standard procedure, calculations are made under the assumption of linear elastic behavior of the material. However, if the RS level is high, then plastic deformations caused by the stress concentration induced by the hole could lead to a stress calculation error. Unlike strain-gage holedrilling (SG-HD) method which records strains only by three (six) strain gauges and elaborates in order to find the RS, the ESPI-HD method allows taking into account the displacements data at all points of the surface around the hole. Analysis of the displacements' variation across the circumference around the hole reveals local features related with plastic deformations. In this paper, a new approach for determination of RS by the hole drilling method that considers the plastic deformations has been proposed. This approach assumes determination of the plasticity effect by calculating stresses using displacements data at various sectors relative to the hole. Analysis of stresses variations calculated using values of displacements at different sectors allows retrieving the real stress state.
\end{abstract}

\section{Introduction}

One of the most widely used technique for determination of residual stresses (RS) is the strain-gage hole-drilling method (SG-HD method). There is an international standard [1] for determination of RS that is based on drilling of a small hole on the materials surface and measuring the surface strains using a special strain gauge rosette attached concentrically around the drilled hole. This method is applied in cases where material behavior is linear-elastic and plasticity effects are neglected. It is known that a hole is the stress concentrator that can result in significant plastic deformations around the drilled holes, if the nearby RS are high. It was shown that if the stressed state does not exceeds about $60 \%$ of the material yield stress $\sigma_{02}$ the errors induced by yielding around the hole are less than $3 \%$ but the plasticity error increases with increasing RS $[2,3]$. This is connected with the fact, that elastic deformations, which for stresses should be calculated, are added to unknown plastic deformation. It is known that deformation data at the three points measured around the hole are not enough to determine a presence or absence of plastic deformation. In the paper [3] the use of a fourelement strain-gauge rosette has been considered to find "effect of plasticity" and real values of RS. Another extension of the hole drilling method is application of a non-contact speckle-interferometry technique to measure displacements around the hole [4-6]. The ESPI-HD method possesses such an important advantage as possibility of displacement data obtaining at a large amount of points near the hole. The analysis of the data allows determining of a presence of plastic deformation around the drilled hole, and to develop a new approach for RS measuring taking into account plastic deformations. 


\section{Determination of RS by the ESPI-HD method}

The ESPI-HD method, similarly to the SG-HD method, assumes measure of displacements at the distance $2.5 r_{0}\left(r_{0}\right.$ - is hole radius $)$ of displacements $u_{x}$. The displacements data are connected with stresses by the following equation [2]:

$$
u_{x}(\theta)=F(\theta) \sigma_{x x}+G(\theta) \sigma_{y y}+H(\theta) \tau_{x y}
$$

where - $F(\theta), G(\theta)$ and $H(\theta)$ - are the functions, which depend on geometric parameters of a hole and mechanical constants of the material, angle $\theta$ determines the coordinates $\left(x=2.5 r_{0} \cos (\theta)\right.$; $\left.y=2.5 r_{0} \sin (\theta)\right)$ of the point, where the displacement $u_{x}$ is measured.

Having the displacements data at the three points $u_{x}\left(2.5 r_{0}, \theta_{1}\right), u_{x}\left(2.5 r_{0}, \theta_{2}\right)$ and $u_{x}\left(2.5 r_{0}, \theta_{3}\right)$, located on a circle with a center at the drilling point, it is possible to compose the system of linear equations (1), and to find $\sigma_{x x}, \sigma_{y y}$ and $\tau_{x y}$. Using the displacements data $u_{x}$ of more than hundred points, unknown values of stresses $\sigma_{x x}, \sigma_{y y}$ and $\tau_{x y}$ are calculated by the least-squares method.

Mathematical simulation with the finite element method was conducted for estimation of plastic deformations influence on the accuracy of RS determination by the ESPI-HD method (Fig.1). As a result of simulation the values of displacements of material surface points depending on the level of stresses in the object were calculated. Then the displacement $u_{x}\left(2.5 r_{0}, \theta\right)$ were selected at the points of surface, located at the distance $2.5 r_{0}$ from the hole center. After that the values of the stresses $\sigma_{x x}$, $\sigma_{y y}$ and $\tau_{x y}$ were found using equations (1). The finite element mesh used by FEM-simulation is presented at the Fig.1. For simulation the following data were used: diameter and depth of hole is 1 $\mathrm{mm}$, object thickness is $4 \mathrm{~mm}$. The area of plastic deformation is highlighted by red color in case when the predefined residual stresses $\sigma_{r s}=0.85 \sigma_{02}$.
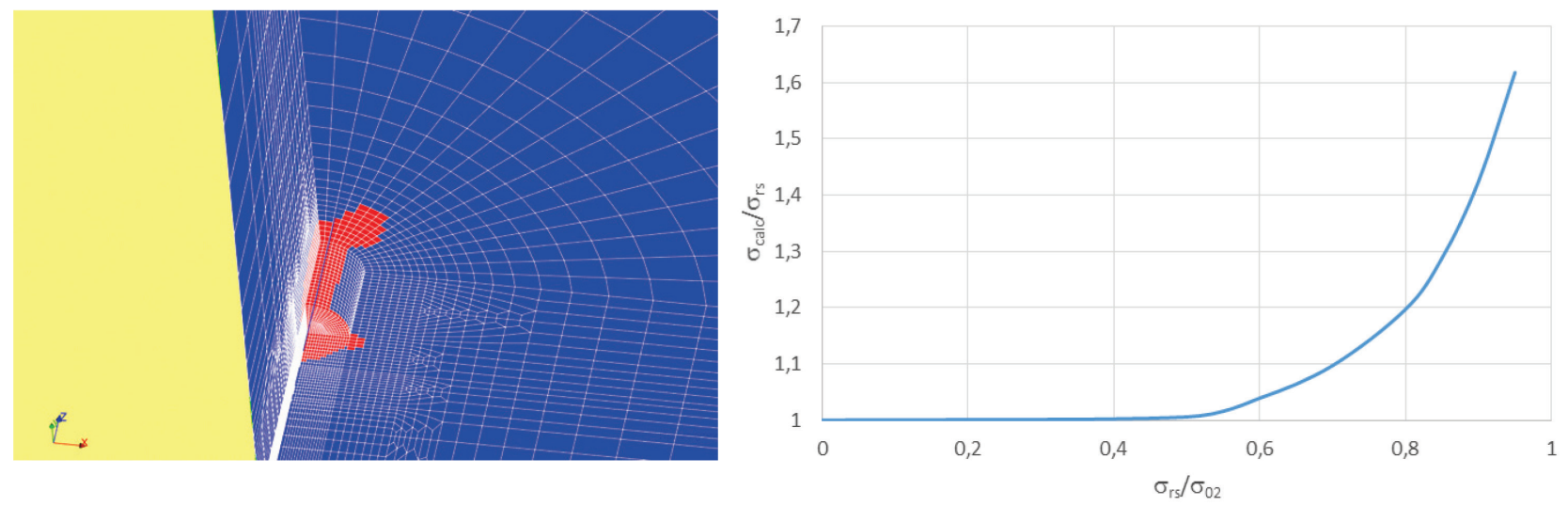

Fig. 1. Finite element mesh used for FE modeling of drilling processes

Fig. 2. Influence of the ratio $\sigma_{r s} / \sigma_{02}$ on the accuracy of $R S$ determination by the ESPI-HD method

To calculate the error in the determination of RS, depending on the level of stresses related to the material yield strength $\left(\sigma_{r s} / \sigma_{02}\right)$, the following numeral experiments were held. The displacements $u_{x, \text { calc }}\left(2.5 r_{0}, \theta\right)$ on the distance $2.5 r_{0}$ for angles $\theta$ in the range $[0,2 \pi]$ were calculated for given ratio $\sigma_{r s} / \sigma_{02}$. Then the system of equations (1) was composed and the values $\sigma_{x x, c a l c}$ were calculated by the least-squares method. It was shown, that at the ratios $\sigma_{r s} / \sigma_{02}>0.6$, the difference between $\sigma_{r s}$ and $\sigma_{x x, c a l c}$ increases, and could reach $60 \%$ (Fig. 2). The area where plastic deformation occurs enlarges with increasing of RS. That results in higher error of RS determination by the ESPI-HD method, which assumes only elastic deformation (elastic stress relaxation) after drilling a hole. Thus, it is necessary to take into account influence of plastic deformation in the calculation of RS. 


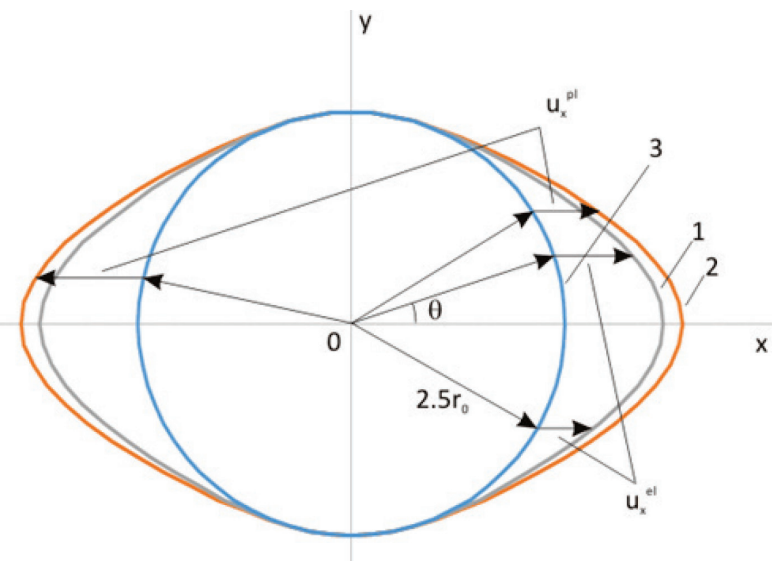

Fig. 3. Geometrical presentation of displacement $u_{x}$, calculated by FEM along the circumference of the radius $2.5 r_{0}$ with the center at the point of drilling, from the angle $\theta: 1$ - linear elastic model; 2 - elasto-plastic model; 3 - circle with radius $2.5 r_{0} .\left(\sigma_{r s}=\right.$ $\left.0.85 \sigma_{02}\right)$.

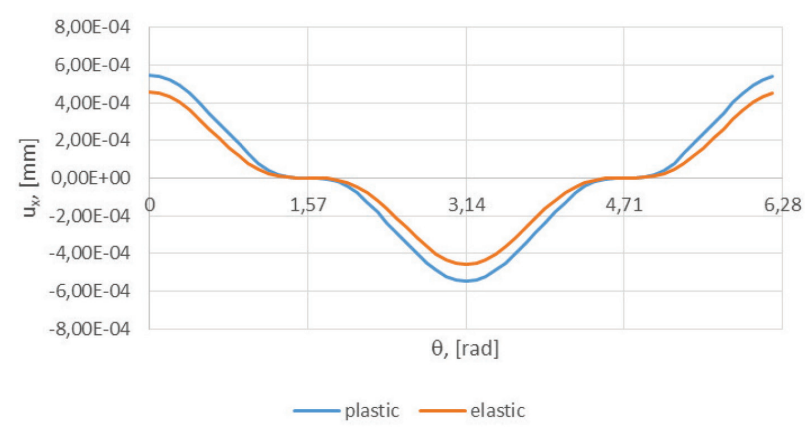

a)

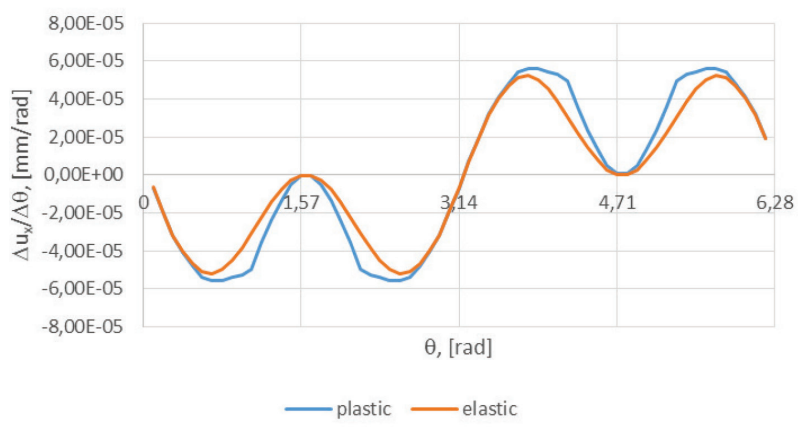

b)

Fig. 4. Dependency of displacements $u_{x}(a)$ and their derivatives $\Delta u_{x} / \Delta \theta$ (b) from the angle $\theta$, along the circumference with radius $2.5 r_{0}$ centered at the point of drilling calculated using the FEM with linear-elastic and elasto-plastic models $\left(\sigma_{r s}=0.85 \sigma_{02}\right)$.

Consider influence of plasticity effect on the results of RS calculation providing that $\sigma_{r s}=$ $0.85 \sigma_{02}$. Geometrical presentation of displacement values $u_{x}$ is shown in Fig. 3. The displacements $u_{x}$ are set relatively from initial position of the point in a larger scale. The displacement curve $u_{x}^{p l}(\theta)$ calculated using elasto-plastic model is similar to the curve $u_{x}^{e l}(\theta)$, that received at linear-elastic model (Fig.4, a). However, derivatives $\Delta u_{x} / \Delta \theta$ (Fig.4.b) calculated under the assumption of linear elastic and elasto-plastic behavior of the material, show that at the angles $\theta$ in the range $[0, \pi / 6]$ the curves $\Delta u_{x}^{p l} / \Delta \theta$ and $\Delta u_{x}^{e l} / \Delta \theta$ are practically identical, and the greatest deviations are observed at $\theta=\pi / 3$. It can be concluded from this graph that the plastic deformations make the greatest influence on the displacements $u_{x}$ of surface points around the hole for the angles $\theta$ in the range $[\pi / 4, \pi / 2]$. Consequently, plastic deformations near the drilled holes increase maximum values of measured displacements, but its contribution is not uniform along the circumference: on separate ranges of $\theta$ dependences of displacements $u_{x}^{e l}(\theta)$ and $u_{x}^{p l}(\theta)$ fit within a constant summand, and on the other ranges they differ the more, than higher the ratio $\sigma_{r s} / \sigma_{02}$. Analysis of distribution of $u_{x}$ along the entire circumference allows identifying presence of plastic deformations and their values.

The feature of the ESPI-HD method is an ability to calculate stresses using the displacement data $u_{x}$ at points of the arc BC that could be chosen arbitrarily around the drilled hole (Fig. 5). Variation of size (defined by the angle $\psi$ ) and position (defined by the angle $\alpha$ ) of the circular arc $\mathrm{BC}$ allows to develop a new technique for determining RS, which takes into account the plastic deformations. The arc is defined by the following way. Some point A with polar coordinates $\left(2.5 r_{0}, \alpha\right)$ is marked on the circle. Regarding this point, the arc $\mathrm{BC}$ that includes all points with angular coordinates from $\alpha-\psi$ 
up to $\alpha+\psi$ is chosen. The displacement $u_{x}$ on arc BC are used for calculation of stresses $\sigma_{x x, \text { calc }}(\alpha, \psi)$ from equations (1).

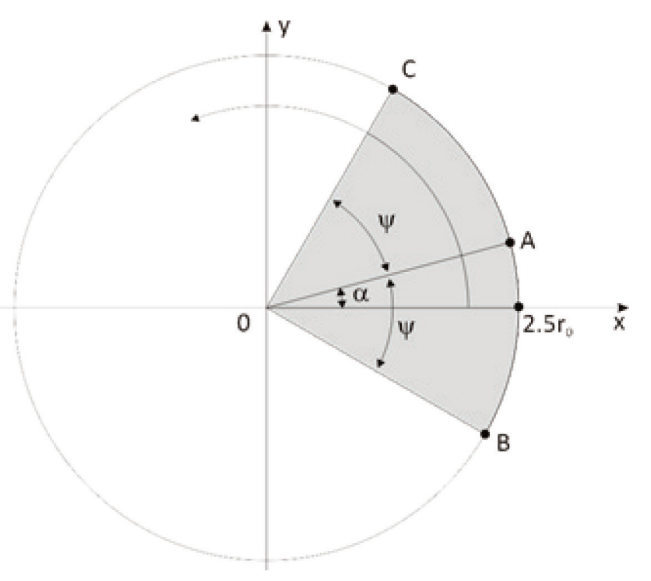

Fig. 5. Location of the arc $B C$ relative to the drilled hole.

Dependences of stress values $\sigma_{x x, \text { calc }}(\alpha, \psi)$, that are calculated using the displacements data measured on the arc BC, on an angle $\alpha$ are presented in the polar (Fig.6, a) and Cartesian (Fig. 6, b) coordinate systems provided that $\sigma_{r s}=0.85 \sigma_{02}$. It is shown that decreasing the length of arc BC (lower values of $\psi$ ) leads to increasing $\Delta \sigma_{\text {diff }}(\psi)$ in the presence of plastic deformation, where

$$
\Delta \sigma_{\text {diff }}(\psi)=\max _{0 \leq \alpha<2 \pi}\left|\sigma_{x x, \text { calc }}(\alpha, \psi)\right|+\min _{0 \leq \alpha<2 \pi}\left|\sigma_{x x, \text { calc }}(\alpha, \psi)\right|
$$

These graphs show significant increase in the difference between the extreme and the averaged value of the stress $\sigma_{x x, \text { calc }}$ at decrease of the angle $\psi$, that is consequence of localization of areas with plastic deformation. One of the parameters that may characterize the plasticity effect is $P E_{\text {diff }}$ :

$$
P E_{\text {diff }}(\psi)=\sqrt{\Delta \sigma_{\text {diff }}(\psi) / \sigma_{x x, c a l c}(\pi)}
$$

Dependency $P E_{\text {diff }}$ on $1 / \psi$ at different ratios $\sigma_{r s} / \sigma_{02}$ is shown in Fig. 7. It is clear that $P E_{\text {diff }}$ equal to zero in the absence of plastic deformation $\left(\sigma_{r s} / \sigma_{02}<0.33\right)$. A monotonic increase of $P E_{\text {diff }}$ with increasing $\sigma_{r s} / \sigma_{02}$ was observed. Thus, based on the analysis of the dependency $P E_{\text {diff }}(1 / \psi)$, we can draw a conclusion about the absence or presence of plastic deformation after drilling a hole in the object. It should be noted that dependencies $P E_{\text {diff }}(1 / \psi)$ obtained for different ratios $\sigma_{r s} / \sigma_{02}$, up to a factor coincide with each other. The figure shows that the ratio $\sigma_{r s} / \sigma_{02}$ is uniquely determined by the slope $k_{\psi}$ of the graph $P E_{\text {diff }}(1 / \psi)$.

The developed ESPI-HD method for determination of RS taking into account the plasticity effects includes the following stages:

1. To calculate values of stresses $\sigma_{x x, \text { calc }}(\pi)$ by equations (1) using the displacements data along a circle with the radius $2.5 r_{0}$ with the center in the point of a drilled hole.

2. For the set of values $\psi$ in the range $[\pi / 6, \pi / 2]$ calculate stresses $\sigma_{x x, \text { calc }}(\alpha, \psi), \alpha=[0,2 \pi]$. For each value of $\psi$ to calculate $P E_{\text {diff }}$. To build a curve $P E_{\text {diff }}(1 / \psi)$. To determine the slope $k_{\psi}$ of the curve.

3. To compute dependency of the slope $k_{\psi}$ on $\sigma_{r s} / \sigma_{02}$ for present experiment conditions (diameter and depth of the hole, thickness of the object, mechanical properties of the material) using FE simulation. To determine $\sigma_{r s} / \sigma_{02}$ and $\sigma_{r s}$, substituting value $k_{\psi}$ calculated in stage 2 into the dependency $k_{\psi}\left(\sigma_{r s} / \sigma_{02}\right)$. 


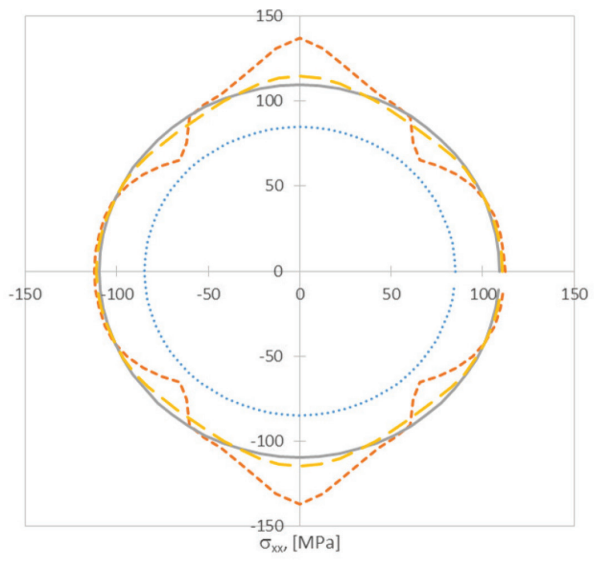

a)

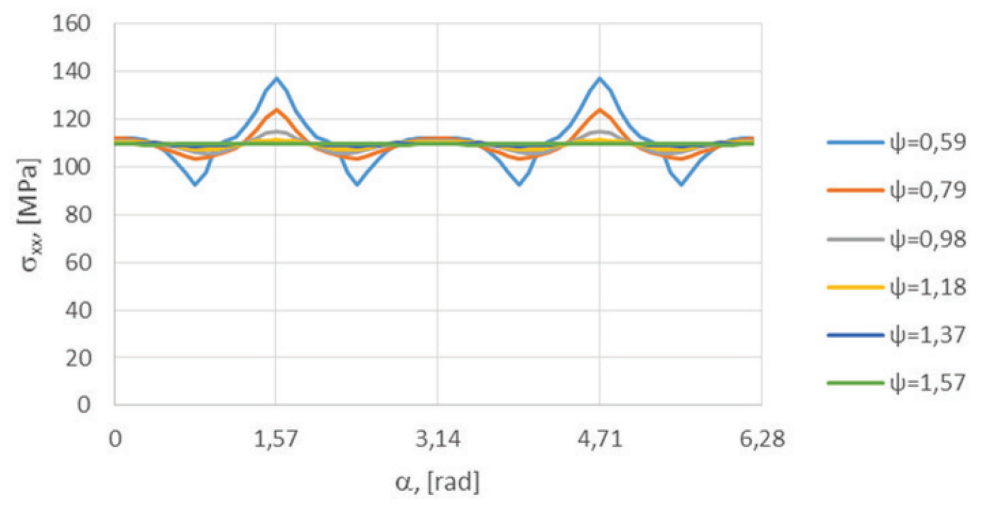

b)

Fig. 6. Dependencies $\sigma_{x x, \text { calc }}$ on $\alpha$ calculated by FEM for different values $\psi$ providing that $\sigma_{r s}=$ $\left.0.85 \sigma_{02}: a\right)$-stress diagram in the polar coordinate system, where gray solid line is stresses $\sigma_{x x, \text { calc }}(\alpha, \psi=\pi)$, orange dashed line is stresses $\sigma_{x x, c a l c}(\alpha, \psi=\pi / 2)$, red dashed line is stresses $\left.\sigma_{x x, \text { calc }}(\alpha, \psi=\pi / 4) ; b\right)$ - in the Cartesian coordinate system.

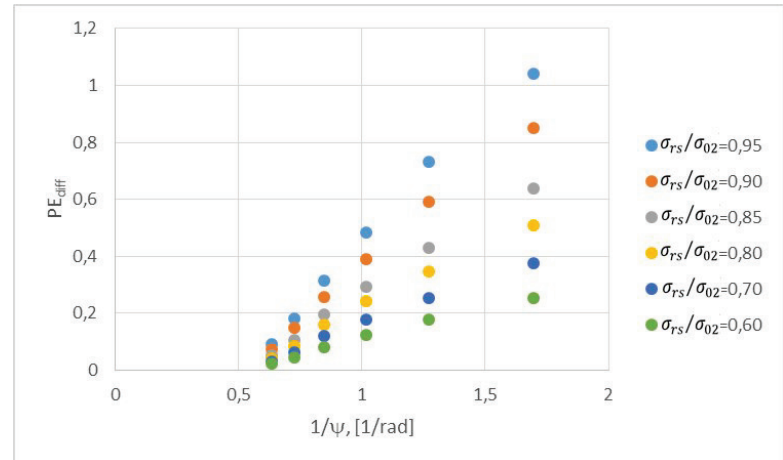

\section{Determination of RS by the ESPI-HD method considering the plasticity effect in welded samples}

RS have been measured by the developed ESPI-HD method considering the plasticity effect in the welded joint made from aluminum alloy (Fig. 8). It is known that in heat-affected zone RS exceed $0.7 \sigma_{02}$ of the material. A compact speckle-interferometer (Fig. 8, a) has been designed for determination of RS. The recorded interference fringe pattern is shown on Fig. 8, b. Values of $\sigma_{x x, \text { calc }}(\alpha, \psi)$ and $P E_{\text {diff }}$ were calculated according to the stage 2 using the displacement data $u_{x}$ in the area marked in blue (fig. 8, b), and as a result the $P E_{\text {diff }}(1 / \psi)$ has been found (Fig. 8, c, d). These graphs are similar to those obtained by mathematical modelling (Fig. 6, 7) and they show presence of significant plastic deformations around the hole. FE simulation has shown that the slope $k_{\psi}$ of the curve (Fig.8, d) fit values of the ratio $\sigma_{r s} / \sigma_{02}=0.92$. Thus, in the point of drilling the residual stresses $\sigma_{x x}=169 M P a$.

\section{Summary}

A new approach for evaluation of the plasticity effect after drilling a hole in the objects being under high residual stresses $\left(>0.6 \sigma_{02}\right)$ is developed. This is achieved by analysis of the displacements data in the points around drilled holes that are measured by the ESPI method. The procedure for calculation of RS with plasticity effects has been proposed. Effectiveness of the ESPI-HD method for measuring of RS at the object in the area of high stress state has been demonstrated. 


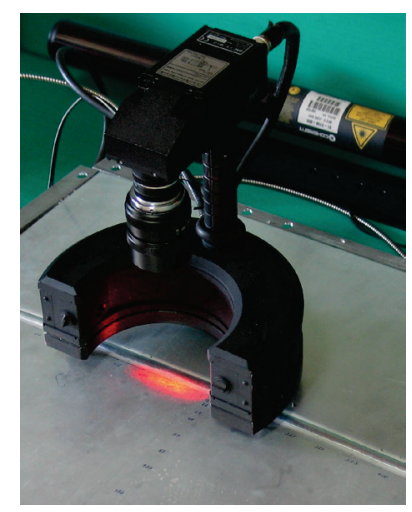

a)

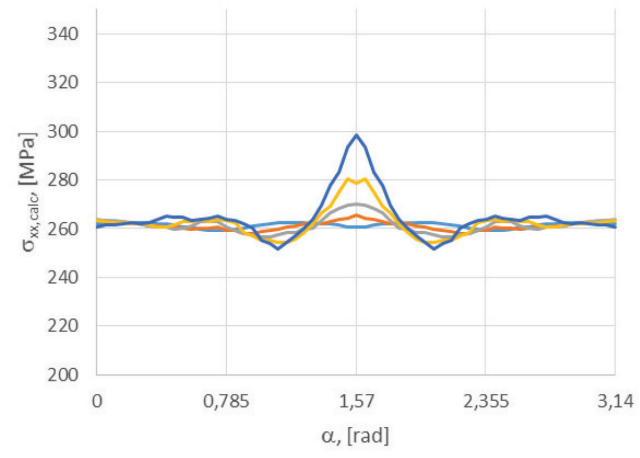

c)

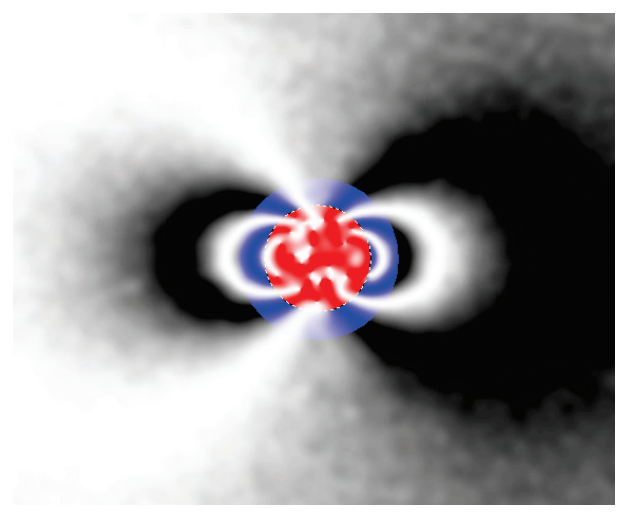

b)

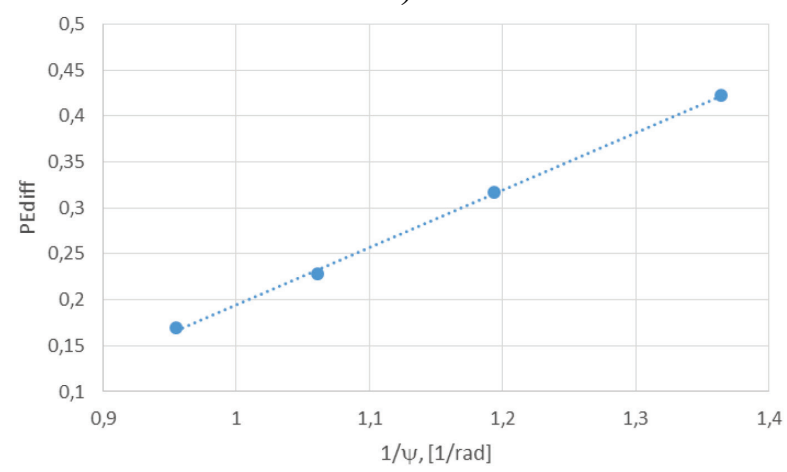

d)

Fig.8. Determination of RS by the ESPI-HD method taking into account the plasticity effect: a) the ESPI-device for determination of RS installed on the welded specimen; $b)$-interference fringe pattern recorded by ESPI after drilling of the hole in the sample; c)-dependence $\sigma_{x x, c a l c}$ on $\alpha$ for different values of $\psi ; d)$-dependence $P E_{\text {diff }}$ on $1 / \psi$.

\section{References}

[1] ASTM E837-13a, "Standard test method for determining residual stresses by the hole-drilling strain-gage method," in Annual Book of ASTM Standards, Am. Soc. Test. Mat. (2013).

[2] Lin YC, Chou CP. Error induced by local yielding around hole in hole drilling method for measuring residual stress of materials. Journal of Materials Science and Technology 1995;11:600-4. http://dx.doi.org/10.1179/mst.1995.11.6.600

[3] D. Vangi and S. Tellini Hole-Drilling Strain-Gauge Method: Residual Stress Measurement With Plasticity Effects J. Eng. Mater. Technol 132(1), 2009.

[4] L.M. Lobanov, V.A. Pivtorak, V.V. Savitsky, G.I. Tkachuk Procedure for determination of residual stresses in welded joints and structural elements using electron speckle-interferometry/ The Paton Welding Journal. 1/2006, pp. 24-29.

[5] M. R. Viotti, W. Kapp and A. Albertazzi Jr., "Achromatic digital speckle pattern interferometer with constant radial in-plane sensitivity by using a diffractive optical element," App. Opt. 48(12), 2275-2281 (2009). http://dx.doi.org/10.1364/AO.48.002275

[6] L. Lobanov, V. Pivtorak, V. Savitsky, G. Tkachuk Technology and Equipment for Determination of Residual Stresses in Welded Structures Based on the Application of Electron Speckle-Interferometry. Materials Science Forum, -2014, Vol. 768, pp. 166-173. 